\title{
Sleepy Heart and Hearty Sleep: Sleep Disorders and Cardiovascular Diseases
}

\author{
V. Gopichandran, S. Suganya and K. P. Misra
}

Department of Cardiology, Apollo Hospitals, Chennai

\begin{abstract}
Sleep is an active, energizing and blissful activity, which everybody enjoys. Diseases affecting any system of the body can alter sleep and sleep disorders can lead to problems in multiple systems of the body. Much research is going on in the field of sleep medicine and its effects on health. The association between sleep disorders and cardiovascular diseases is well known. O bstructive Sleep Apnea (OSA) is known to cause ischemic heart disease and other related cardiovascular diseases. Severe heart diseases like congestive heart failure also are known to produce sleep disordered breathing. Relationship of cardiovascular diseases to certain phases of the sleep cycles has also been reported. There is a definite need for physicians, cardiologists and sleep specialists to work together in this field of sleep disorders and cardiovascular diseases in order to improve detection and management of these conditions.
\end{abstract}

Indian J Sleep Med 2006; 1.3, 141-144

\section{Introduction}

Sleep is a blissful state, which allows one to rejuvenate and prepare for the day ahead. $O$ ver the years more and more has been understood about the essential nature of sleep. Sleep is essential for consolidation of memory and for coordination of several important metabolic processes. Sleep deprivation for more than 10 days can even be fatal. Sleep medicine is slowly emerging as an important and essential specialty. Sleep specialists are starting to work in coordination with other specialists to provide good sleep health to the patients. A close association has been established between sleep disorders and cardiovascular diseases. O bstructive Sleep Apnea (OSA) has been known to be associated with ischemic heart disease and its related conditions. Similarly chronic heart failure is known to be associated with central sleep apnea. Some cardiac diseases have more propensity to

Address for correspondence:

\section{Dr. K.P. Misra}

Senior Consultant Cardiologist

Apollo Hospitals, Chennai 600006

E Mail - misra_k_p@yahoo.com occur during specific phases of sleep cycle. This paper will look into the association between sleep disorders and cardiovascular diseases. D o patients who have a sleepy heart have a hearty sleep? Are patients who do not have a hearty sleep prone to have sleepy hearts? This paper will try to answer these questions.

\section{Cardiovascular Physiology in Sleep}

Sleep is a dynamic and active process unlike what appears to the unknowing mind. There are many specialized changes that take place during sleep. The most important among these changes are the cardiovascular system alterations. During REM sleep there is an increased neurological activity in the whole of the brain. When this activity passes through the parts of the nervous system that control the cardiovascular system, instability in cardiovascular function occurs. The cardiovascular regulation during REM sleep is erratic. During REM sleep increased heart rate, increased blood pressure and rapid fluctuations in the blood pressure have all been documented. D uring the Phase 3 and Phase 4 of N REM sleep the cardiovascular system is more regulated. Therefore physiologically the cardiovascular system

Indian Journal of Sleep M edicine (IJSM ), Vol. 1, N o. 3, 2006 
during sleep goes through phases of hyper and hypo activity ${ }^{1}$.

The relationship between sleep and cardiovascular system regulation is evident by the increased propensity of developing certain cardiovascular diseases during certain times of the day. For example, 20 percent of myocardial infarctions, 15 percent of sudden deaths and 29 percent of episodes of atrial fibrillation occur between midnight and $6 \mathrm{AM}^{1}$. This has variably been attributed to the circadian variation of secretion of hormones and the dynamic cardiovascular changes that occur during sleep. M ark Twain, in a lighter vein, once commented, "M ost people die in the bed during sleep. I therefore avoid going to bed." Therefore there seems to be an interesting relationship between sleep and cardiovascular diseases.

\section{Sleep Diseases leading to Cardiovascular Disorders}

The prototype of a sleep disorder that leads to cardiovascular disease is OSA. OSA is defined as repetitive upper airway obstructions during sleep resulting in profound effects on cardiovascular function and gas exchange.

\section{Acute Cardiovascular Changes in OSA}

OSA is characterized by phases of hypoxemia, reoxygenation, hypercapnea, repetitive intrathoracic pressure changes and arousals from sleep. This in turn leads to sympathetic activation causing increased levels of circulating catecholamines, tachycardia, vasoconstriction and impaired cardiovascular variability? Autonomic system changes during apnea cause heart rate to reduce and when the breathing resumes heart rate speeds. It has been shown that $80 \%$ of O SA have rhythm abnormalities, $18 \%$ have recurrent sinus pauses, asystole and ventricular extra systoles. In patients with cardiac disease, OSA increases the risk of arrhythmias including dangerous ventricular arrhythmias. Apart from these rhythm and heart rate disturbances there are also significant changes in the blood pressure. Systemic blood pressure increases during apneic episode. In the elderly the systemic blood pressure falls during apnea and the pulmonary artery pressure increases due to hypoxemia and pulmonary vasoconstriction. The repetitive falls in the intrathoracic pressures leads to reduction in stroke volume and total cardiac output. The repetitive arousal from the sleep, which is actually a protective mechanism, has its own detrimental effects on the cardiovascular system. D uring arousal there is a surge in blood pressure and vasoconstriction due to increased sympathetic tone? It was also observed that during the apneic spells in OSA there was a peak in the incidence of ST segment deviations. Apart from these acute changes there are chronic changes that occur with OSA.

\section{Chronic Cardiovascular Changes in Obstructive Sleep Apnea:}

The chronic hypoxia and hypercapnea leads to persistently elevated sympathetic tone in patients with OSA. Endothelin 1 levels have been shown to be raised in these patients ${ }^{3}$. Endothelin 1 being a potent vasoconstrictor causes marked elevation of the blood pressure. Plasma levels of angiotensin II and aldosterone are also elevated in OSA again contributing to increased blood pressure in these patients. Patients with OSA have significantly higher levels of homocysteine than patients without, homocysteine being a non-traditional coronary risk factor ${ }^{4}$. Patient with chronic OSA have also been shown to have vascular oxidative stress ${ }^{5}$. M arkers of inflammation in these patients are elevated ${ }^{6}$, as are the pro-coagulation factors such as PAI $1^{7}$. Patients with OSA frequently have metabolic dysregulation indicated by obesity, insulin resistance and leptin resistanceall of them contributing to cardiovascular system abnormalities ${ }^{8}$.

\section{Cardiovascular Diseases in Obstructive Sleep Apnea}

Based on the above-discussed changes, which are observed in patients with OSA, they develop thefollowing cardiovascular diseases:

- Systemic hypertension - sometimes refractory to treatment.

口 Pulmonary hypertension

a Cor pulmonale

Ischemic heart disease

a $\mathrm{H}$ eart failure

Stroke

In one study of patients with different cardiovascular diseases it was found that OSA was present in $60 \%$ of 
patients with stroke, $80 \%$ of drug resistant hypertension, $50 \%$ of congestive heart failure, $30 \%$ of all coronary artery disease ${ }^{1}$. The specific relationship with hypertension and OSA was established in one study in which in every age group people who were snorers had statistically significant higher prevalence of hypertension. The Sleep H eart H ealth Study studied about 6500 patients who underwent a polysomnography. $16 \%$ of them reported cardiovascular disease ( $\mathrm{MI}$, angina, $\mathrm{PCl}$, $C A B G$, stroke). O dds of OSA being associated with cardiovascular diseases were 1.42, which was quite significant ${ }^{9}$. M oreover it was also found that if the OSA was identified and treated, the cardiovascular diseases improved.

Treatment of the OSA is very important in these patients. The principle of treatment is reduction of risk factors, treatment of any anatomical cause for the OSA and if no cause is found assisted ventilation techniques to relieve the obstruction. Weight loss, avoidance of alcohol and sedatives before going to bed, and procedures such as tonsillectomy, uvuloplasty and adenoidectomy if indicated are simple measures. The ventilatory assistance methods are Continuous Positive Airway Pressure (CPAP) and Biphasic Positive Airway Pressure (BiPAP). They are the most widely used treatments and the treatment of first choice in patients with obstructive sleep apnea syndrome. The principle of these devices is that the positive pressure splints the pharyngeal airway open, counteracting its tendency to collapse during inspiration. Treatment with nasal CPAP has been shown to improve quality of life, cognitive function, and arterial hypertension ${ }^{10,11,12}$.

\section{Cardiovascular Disorders leading to Sleep Diseases}

Patients with $\mathrm{C}$ ongestive $\mathrm{H}$ eart Failure have been shown to suffer from central sleep apnea. In 1818, Cheyne first described repetitive cycles of apnea followed by hyperpnoea in what is now called Cheyne-Stokes respiration. It is most often linked to left heart failure. It has also been observed in other conditions such as after some cerebrovascular accidents, renal disease, and exposure to high altitude. $40 \%$ of all patients with left ventricular ejection fraction less then $45 \%$ have Cheyne Stokes breathing ${ }^{13}$. The risk factors for Cheyne Stokes breathing in congestive heart failure include male gender, age $>60$ years, atrial fibrillation and hypocapnea.

\section{Mechanism of Central Sleep Apnea in Patients with Congestive Heart Failure}

In congestive heart failurethere is a heightened ventilatory response to hypercapnea, alkalemia, sluggish circulation and the sleep state. These act as the trigger and they induce hyperventilation. This causes carbon-dioxide wash out and hypocapnea. This is sensed by the respiratory center rather late due to sluggish circulation and after someextended time of hyperventilation it produces apnea. After a period of apnea a cycle of hyperventilation is triggered and this cycle perpetuates itself. The important factor is the sluggish circulation. D uring these cycles of abnormal breathing there is worsening of the heart failure state due to raised sympathetic system activity and variations in the myocardial oxygen demand and supply. Patients with central sleep apnea have been observed to have sleep onset type of insomnia, recurrent arousal, hypoxemia, fluctuating blood pressure and episodes of paroxysmal nocturnal dyspnea ${ }^{14}$. It was postulated that treatment of this central sleep apnea with positive airway pressure ventilation during sleep would improve the cardiac status. In the $C$ anadian Positive Airway Pressure (CAN PAP) Study patients with heart failure and central sleep apnea were randomly assigned to receive either continuous positive airway pressure (CPAP) or no treatment in order to test whether intervention improved transplant-free survival. Treatment of central sleep apnea in the CAN PAP trial resulted in lower norepinephrine levels, a slightly increased ejection fraction, and an early but transient improvement in exercisetolerance. But there was no definite mortality ben efit ${ }^{15}$. The question now is whether the central sleep apnea really contributes to worsening of the heart failure or if the treatment of the heart failure is sufficient.

\section{Condusion}

Thus it is clear that there are conditions where an abnormal sleep disturbance can lead to heart disease and indeed people who do not have a hearty sleep have the risk of having a sleepy heart. Similarly people with heart failure have the risk of disturbed sleep; people with a sleepy heart indeed miss out on a hearty sleep. It is essential to recognize this association and take remedial measures to manage the heart health and sleep health.

Indian Journal of Sleep M edicine (IJSM ), Vol. 1, N o. 3, 2006 


\section{References}

1. Verrier R: Sleep-related cardiovascular risk. In Kryger M Roth T, Dement WC: Principles and Practice of Sleep Medicine. 4th ed. Philadelphia, WB Saunders, 2004

2. Peled N, Greenberg A, Pillar G, et al. Contributions of hypoxia and respiratory disturbance index to sympathetic activation and blood pressure in obstructive sleep apnea syndrome. Am J Hypertens 1998; 11:1284-1289.

3. Ip M.S., Tse H.F., Lam B. Endothelial function in obstructive sleep apnea and response to treatment. Am J Respir Crit Care Med (2004) 169: pp 348-353.

4. Lavie L, Perelman A, Lavie P. Plasma homocysteine levels in obstructive sleep apnea: association with cardiovascular morbidity. Chest 2001; 120:900-908

5. Dugovskaya L, Lavie L, et al. Activation of oxidative metabolism and CD64 and CD11c expression in peripheral blood monocytes and neutrophils in obstructive sleep apnea syndrome. Am J Respir Crit Care Med 2002; 165:934939

6 Jialal I, Devaraj S, Venugopal SK. C-reactive protein: risk marker or mediator in atherothrombosis? Hypertension 2004; 44:6-11

7. von Kanel R., Dimsdale J.E., Hemostatic alterations in patients with obstructive sleep apnea and the implications for cardiovascular disease. Chest (2003) 124 : 1956 1967.

8. Ip M.S., Lam B., Ng M.M.. Obstructive sleep apnea is independently associated with insulin resistance. Am J Respir Crit Care Med (2002) 165: 670-676.

9 Nieto FJ, Young TB, Lind BK, et al. Association of sleepdisordered breathing, sleep apnea, and hypertension in a large community-based study. Sleep Heart Health Study. JAMA. 2000 Apr 12;283(14):1829-36.

10 Engleman HM, Kingshott RN, Wraith PK, et al: Randomized placebo-controlled crossover trial of continuous positive airway pressure for mild sleep apnea/hypopnea syndrome. Am J Respir Crit Care Med 159:461-467, 1999.

11. Redline S, Adams N, Strauss ME, et al: Improvement of mild sleep-disordered breathing with CPAP compared with conservative therapy. Am J Respir Crit Care Med 157:858865, 1998.

12. Faccenda JF, Mackay TW, Boon NA, Douglas N J Randomized placebo-controlled trial of continuous positive airway pressure on blood pressure in the sleep apneahypopnea syndrome. Am J Respir Crit Care Med 163:344348, 2001.

13. Javaheri S, Parker TJ, Liming JD, et al: Sleep apnea in 81 ambulatory male patients with stable heart failure: Types and their prevalences, consequences, and presentations. Circulation 97:2154-2159, 1998.

14. Javaheri S: A mechanism of central sleep apnea in patients with heart failure. N Engl J Med 341:949-954, 1999.

15. Bradley TD, Logan AG, Kimoff RJ et al. Continuous Positive Airway Pressure for Central Sleep Apnea and Heart Failure. N Engl J Med. 2005 Nov 10;353(19):2025-33. 